\title{
Analysis of Fraudulent Photographs Submitted to Federal Court and the United States Congress
}

\section{Bryan Burnett ${ }^{*}$}

Meixa Tech, Cardiff-by-the-Sea, CA, USA

\begin{abstract}
Photographs or recently digital images are routinely taken during autopsies. The analysis and interpretation of those images assumes that they are properly taken and handled, and can be relied upon. This paper concerns a case in which manipulation of such an image may have led to an unfounded conclusion in a death investigation. In 1994, as a result of the wrongful death civil case in United States District Court Central District of California Southern Division (SA CV 93-991) brought against the Department of Defense by Dr. David Sabow and Sarah Sabow, brother and wife of the victim, United States Marine Colonel James E. Sabow, approximately 30 autopsy photographs were released. One of the autopsy photographs differed from all the other photographs by appearing to be poorly taken. This photograph of the victim's posterior upper body appeared to have had the flash too close to the victim's lower left side, which caused the upper back, neck and left pinna to be overexposed. Digital analysis of a high resolution scan of this photograph revealed it was likely the result of darkroom manipulation. A nearly identical photograph to the questioned photograph, submitted in a report to the United States Congress, was a different fabrication. In December 2012 the original, unmodified photograph was discovered from which both manipulated photographs were generated. The original photograph allowed for a more detailed analysis of the questioned photographs and showed that perjured submissions had occurred: the first in Federal Court and the second to the United States Congress.
\end{abstract}

Keywords: Forensic science; Autopsy photograph; Darkroom manipulation; Imaging processing; Federal court, United states congress

\section{Introduction}

The victim, United States Marine Corps Colonel, James E. Sabow, was found dead in the backyard of his southern California officer quarters on the El Toro MCAS January 1991. The death certificate, issued the next day, declared the victim died by suicide and the autopsy report [1] concluded this was by a self-inflicted intraoral shotgun discharge.

Three sets of autopsy photographs were release at different times during the more than twenty years since the death of Colonel James Sabow:

- Released from the Department of Defense (DOD) in response to a civil case in Federal Court (Sara Sabow et al. v. The United States, US District Court, Central District of California, Southern Division. SA CV 933-991 AHS). Year of release of these photographs will be indicated on the images as "1994."

- Released from the DOD to Dr. Jon Nordby for a report to the US Congress. The report was submitted in 2004 by Jon Nordby [2]. Year of release of these photographs will be indicated on the images as "2004."

- Released from the Orange County Coroner's Office (CA) in December 2012. Year of release of these photographs will be indicated on the images as "2012."

The photographs and X-ray images from 1994 and images of photographs from the 2012 release show the victim sustained a powerful blow to his right occipital skull prior to an intraoral shotgun discharge. An X-ray lateral view (Figure 1A) shows the depressed right occipital fracture. The victim lived long enough after the blow to express additional indicators of a pre-mortem fracturing of his skull, where the occipital fracturing extended to the basal bones which generated the so-called "raccoon eyes" [3,4] (Figure 2B). In addition, there was extraordinary swelling of the right side of the face (Figure 2B) and right neck (Figure 2C), which can only have occurred premortem.

\section{Image Information Loss}

The analyses of the photographs from this case require an understanding of potential information loss with photograph copying by optical means (i.e., a photo enlarger) or optical/digitizing means (i.e., computer controlled scanner). How much information is lost when the original negative (first generation) is copied by optical means (photo enlarger) creating a second generation photograph print? Information loss will always occur, which would be in the form of blurring. The amount of information loss depends on the amount of enlargement, quality of the optics of the duplicating equipment, etc [5]. Scanning of a photograph or negative or some situations where copying a digital image into a different format (e.g., a JPEG image into an Adobe PDF document) could cause a gamma shift for the image [5]. Thus, for each new image copy, a new generation is created. The third or fourth generation images examined in this study not only have this information loss but also have gamma shifts.

The photographic evidence submitted in 1994 was color 8 X 10 -inch glossy prints. Negatives or prints, either copied from the original negatives or the original negatives, were sent to the DOD from the

*Corresponding author: Bryan Burnett, P.O.Box 844, Meixa Tech, Cardiff-by-theSea, CA, USA, Tel: (760) 634-5939; Email: brburnett@meixatech.com

Received August 24, 2016; Accepted October 14, 2016; Published October 17 2016

Citation: Burnett B (2016) Analysis of Fraudulent Photographs Submitted to Federal Court and the United States Congress. J Forensic Res 7: 343. doi: 10.4172/2157-7145.1000343

Copyright: () 2016 Burnett B. This is an open-access article distributed under the terms of the Creative Commons Attribution License, which permits unrestricted use, distribution, and reproduction in any medium, provided the original author and source are credited. 
Citation: Burnett B (2016) Analysis of Fraudulent Photographs Submitted to Federal Court and the United States Congress. J Forensic Res 7: 343. doi: $10.4172 / 2157-7145.1000343$

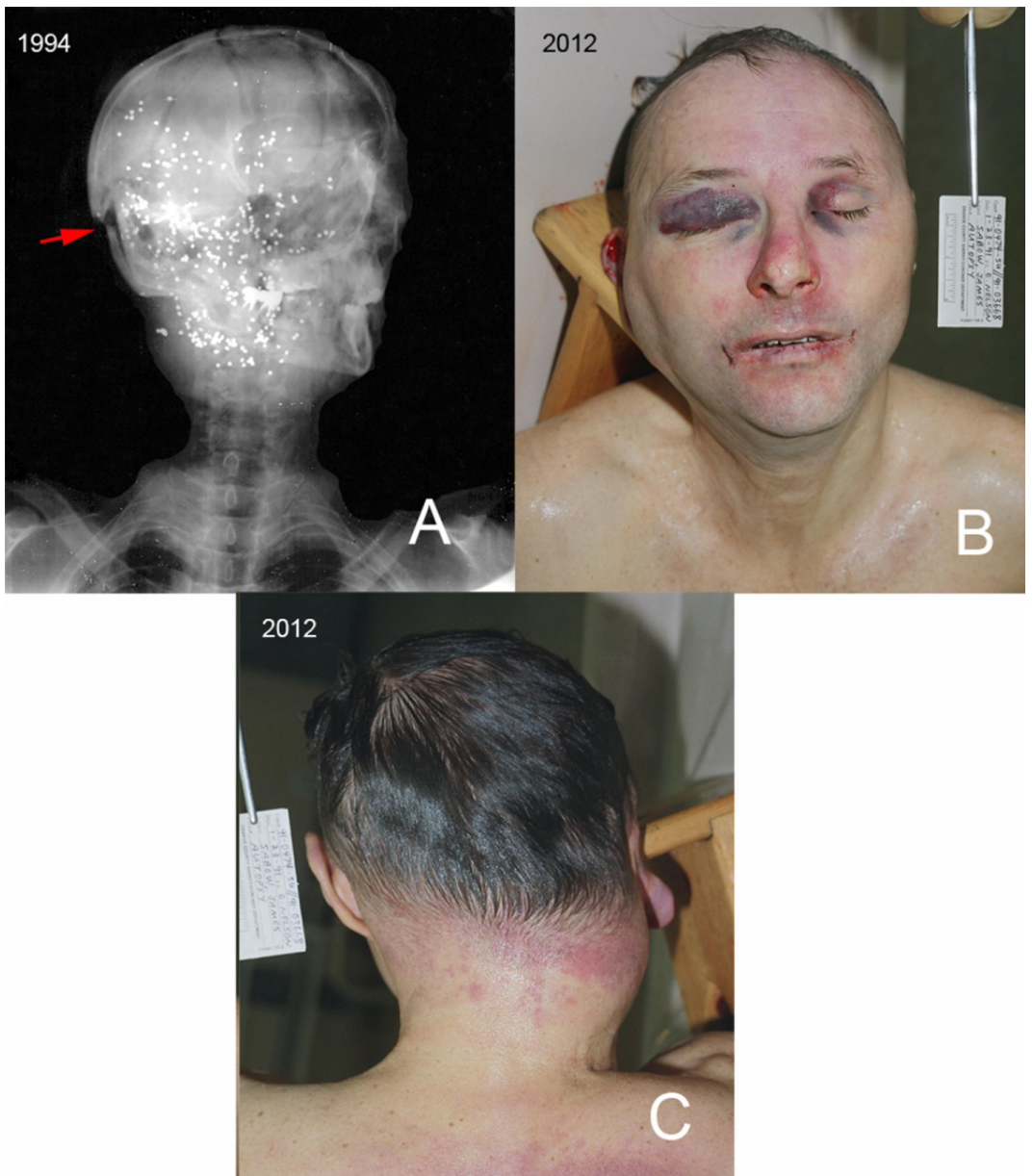

Figure 1: Evidence that the victim received a blow to his right occipital prior to an intraoral shotgun blast; see Sabow and Burnett [6] for a detailed pathology analysis. A. X-ray showing a depress skull fracture, at arrow; the bright spots are the shotgun pellets. B. Preautopsy photograph show swelling on the right side of the Colonel's face; prominent ecchymosis of the right eyelid and preorbital ecchymosis, "raccoon eyes," is indicative of basal skull bone fracture [4] which occurred prior to death. C. Posterior of the victim showing extraordinary swelling of the right neck.

California Orange County Coroner. These photographic prints from the DOD were submitted for the author's analysis.

One of the autopsy photographs (Figure 2) from the 1994 autopsy photograph release was inconsistent with the others by having the appearance of being poorly taken (flash at lower left and too close to the subject). A nearly identical appearing image of the same photograph was submitted in the 2004 report [2] to the United States Congress. The author will show these inconsistent (questioned) photographs were modified from the original photograph.

\section{The first modified photograph}

Many features of the 1994 questioned photograph (Figure 2) suggest doctoring. These disparate features are the large amount of shadow surrounding the victim, the strange illumination of the subject and the absence of swelling of the right distal neck. Other autopsy photographs show prominent swelling in this area (e.g., Figures $1 \mathrm{~B}$ and $1 \mathrm{C}$ ).

\section{Deep shadow information content in color photographs}

The large amount of deep shadow in the questioned photograph suggests an analysis approach: is there hidden detail within the deep shadow in color photographs? The human eye does not have the

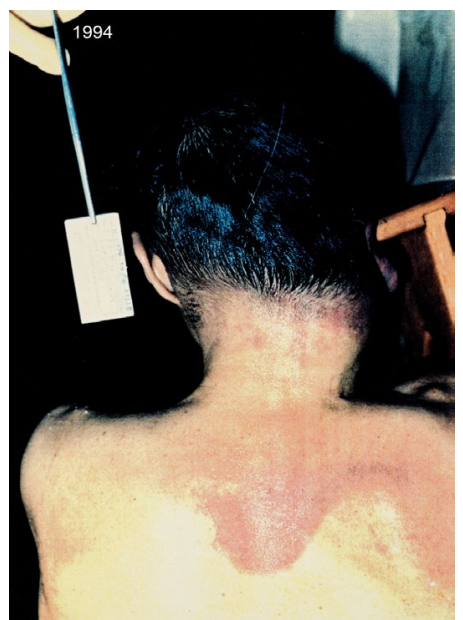

Figure 2: Image of the questioned 1994 photograph; if assumed legitimate; the flash was too close to the subject and to the left causing the subject's upper back and neck appear overexposed and the background and most of the head in deep shadow. The black splotchy area of the distal left neck, just behind the ear, is an ink smear from a Bates stamp transfer. 
Citation: Burnett B (2016) Analysis of Fraudulent Photographs Submitted to Federal Court and the United States Congress. J Forensic Res 7: 343. doi: 10.4172/2157-7145.1000343

Page 3 of 8

sensitivity to discern the full range of colors that are displayed in a 24 bit image. A typical 24 bit color image has more than 16 million possible colors. Our perception only can distinguish between a small fraction of those colors $[5,6]$. Even though we see only black in the deep shadow areas of the questioned photograph could there be information present, but we cannot see it? Can a CCD-based scanner-produced digital image of a photograph retain hidden information in that print photograph's deep shadow, if it exists? This issue needs to be explored before tackling the examination of the deep shadow or black areas of the questioned photograph.

Structures within deep shadow will likely be revealed by image processing. This is done by expanding (increasing gamma) the black pixel areas into visual range. Adobe Photoshop, using its Levels tool (gamma adjust) routine, unseen colors present in a shadow of an image can be "stretched" so that the human eye can perceive these color differences, if they exist.

A $35 \mathrm{~mm}$ negative photograph taken and printed in 1985 shows that its deep shadow areas, when scanned by the author's CCD Epson scanner (model 4180 Photo), has previously unseen structure content when the deep shadow area's hidden color content is expanded by increasing gamma (Figure 3). The conclusion is color photographic prints where there is deep shadow or black can retain information not discernable to the human eye.

Images of the head profile photographs of the victim have deep shadows (Figures $4 \mathrm{~A} 1$ and $4 \mathrm{~B} 1$ ) which when gamma adjusted, reveal structure (Figures $4 \mathrm{~A} 2$ and 4B2). The questioned photograph, if it was printed from the same original negative strip as the other photographs of this autopsy (there is no reason not to assume this), should also have hidden background detail within its deep shadows. Even in a third generation or greater photograph or image where information loss from the first generation negative would be expected, features within deep shadows can be still observed by increasing gamma (e.g., Figure 3). Clipping (setting all color values in a color range to a single color value) of the black areas in a photograph does not occur with normal copying to different formats (e.g., conversion from TIFF to JPEG), but does occur with conversion of images to Adobe Acrobat PDF format (unpublished observation).

\section{The questioned photograph}

From the way the upper body and head are illuminated in the questioned photograph (Figure 5A1) if real, suggests the flash is located lower left of the victim. With the flash in this position, more hair like that at the hair line should be showing. This is simply because the back of the head projects higher than the neck. An attempt was made to duplicate the flash and camera angle of a hair dresser's mannequin head (Figure 5B1) with a Canon 6.3 MP SLR camera. The purpose of this image is to show that the back of the head would have similar illumination from the neck to almost the top of the head and therefore the imaging characteristics of the hair on the back of the head of the victim (Figure 5A1) should be uniform from the neck through at least to the distal part of the occipital region of the head. It is not.

An unusual aspect of the questioned photograph is the large amount of shadow surrounding the victim's head. It is possible to superficially duplicate a deep shadow surround the subject by a positioning of the

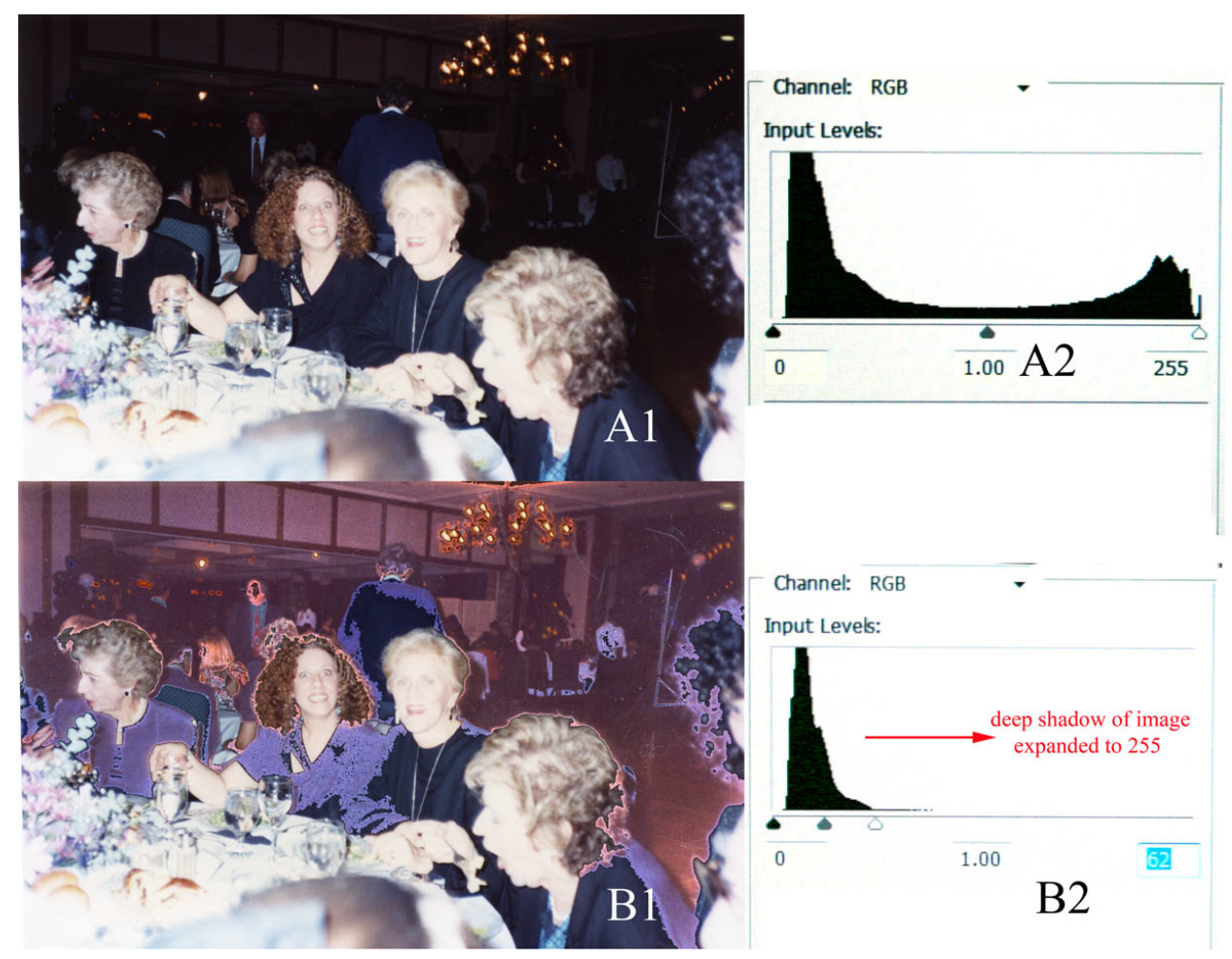

Figure 3: Image of Photograph (24 bit) of revelers at a restaurant overexposed by the camera's flash and the background in deep shadow; from a photograph print circa 1985. A1. The native image (third generation) shows little detail in the deep shadow. A2. Graph generated by Photoshop Levels showing pixel distribution for the A1 image. B1. Shadow and dark clothing segmented in A1 and pixel range (pixel region at left in A2) expanded using Photoshop's Levels. B2. Photoshop's Levels graph shows pixel distribution for the segmented area that was pixel expanded in the A1 image; many features of the room and its occupants are now apparent. 
Citation: Burnett B (2016) Analysis of Fraudulent Photographs Submitted to Federal Court and the United States Congress. J Forensic Res 7: 343. doi: $10.4172 / 2157-7145.1000343$

camera flash (Figure 5B1). As shown with other images of other 1994 autopsy photographs (Figures 4A1 and 4B1), the Photoshop gamma increase (Figures 4A2 and 4B2) of the deep shadow areas reveal details that were hidden. The gamma increase using Photoshop's Levels routine shows the questioned photograph's deep shadow has no structural detail (Figure 5A2), except for horizontal lines or stripes on a variable density purple. This structure within the deep shadow of the questioned photograph actually extends through most of the victim's head region.

The odd transition from the hair at the hair line (Figures 6A1 and 6A2) to the white reflective, probably wet, hair cannot be duplicated by the mannequin imaging (Figure 5B1). Another autopsy photograph of the left side of the head of the victim (Figure 6B1) shows hair reflections similar to the questioned photograph, but the former shows transition (Figure 6B2) to the deep shadow. Such a transition in the questioned photograph (Figure 6A2) is abrupt and the reflective hair which shows white in the image is mixed with the normal-imaged hair at the hair line.

\section{The original photograph}

In December 2012, the Orange County (California) Coroner's facility where the victim's autopsy occurred, released images of the photographs taken during the autopsy. Among these images was one showing the posterior upper body and head (Figure 7A). This newly discovered image is shown with the questioned photograph image

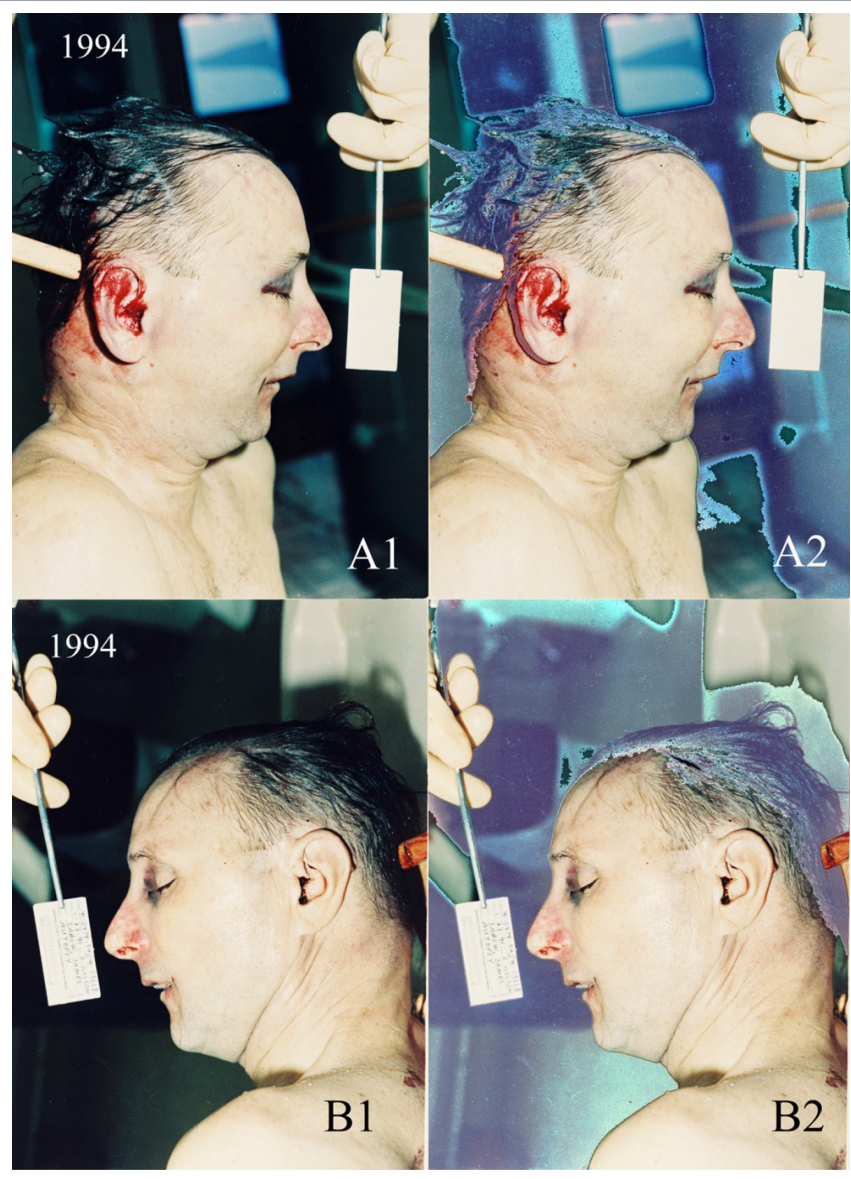

Figure 4: Left and right profiles victim taken during the autopsy (A1 and B1) Deep shadow (some of which could occur due to gamma shift in image copying) is present in both photographs (A2 and B2) Background detail of the shadows are revealed when the deep shadow is isolated and gamma adjusted with the Photoshop Levels routine.
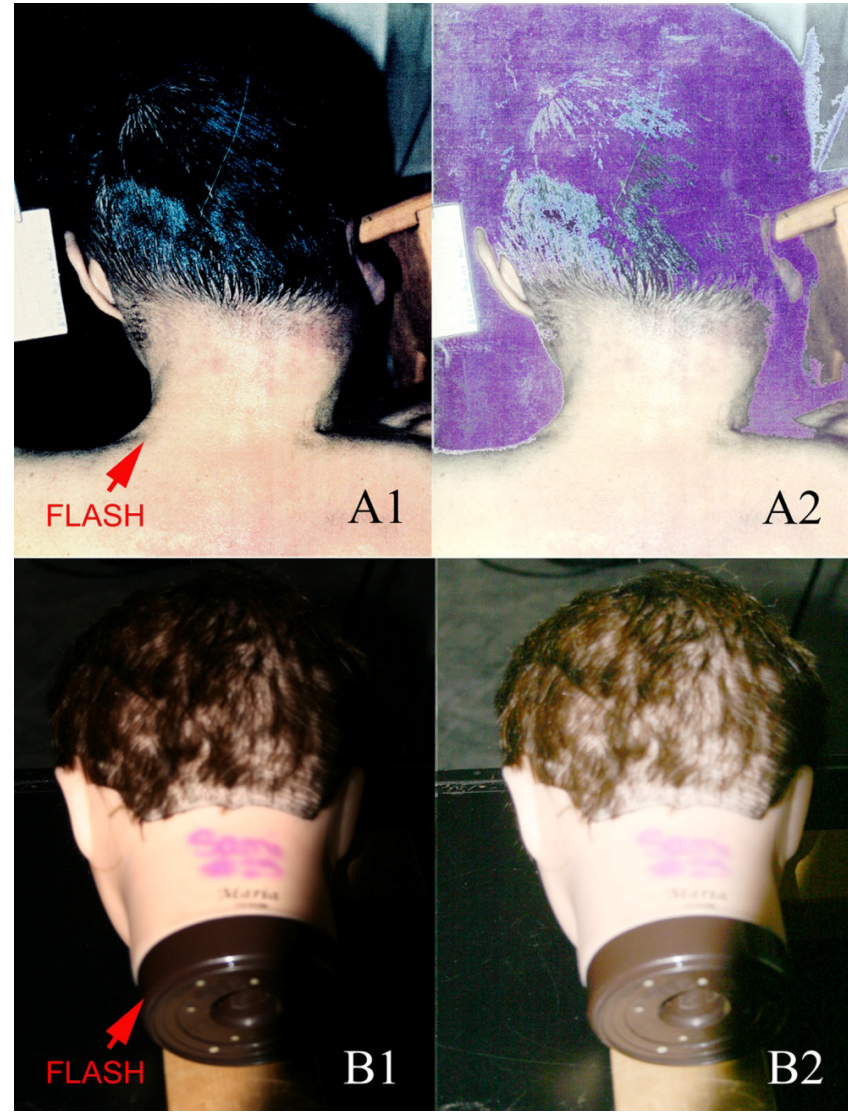

Figure 5: Back of head illumination and gamma adjustment. A1- The questioned photograph for comparison to the mannequin head; the probable flash location and direction is shown. A2- Full image gamma increase which shows no background other than apparent scan lines of stripes (see below) on a base color of purple. B1- Mannequin head with short hair; the angle of the flash and camera lens in the questioned image was attempted to duplicate the lighting for this image as shown in A1. Unlike the head in the questioned image, the illumination of the mannequin hair shows continuous structure and form to nearly the top of the head. The probable flash location and direction is shown. The deep shadow surrounding the mannequin head was naturally produced. B2- Detail of the head that was in the shadow areas brought out by Photoshop's Levels routine; the amount of gamma adjustment was identical to that of $A 2$. The table on which the mannequin head rests is painted black and carpet at the top of the image is gray. Electrical wires are on the carpet.

(Figure 7B). On the basis of simple visual comparison, there is no doubt the questioned photograph was modified from this photograph. How was this accomplished? Was it by an image processing program such as Photoshop, or was it through a photo enlarger using darkroom techniques with airbrushing? The analysis of the questioned photograph indicates the latter.

\section{Evidence of airbrushing}

It is apparent focused modification of the questioned photograph occurred on the right distal neck area (compare this region in the unmodified photograph in Figure $7 \mathrm{~A}$ to the modified photograph, Figure $7 \mathrm{~B}$ ). Figure $8 \mathrm{~A}$ is a photograph that has known airbrush modification within the area outlined. Enlargement of this area in Figure $8 \mathrm{~A}$ is compared to a similarly enlarged area (outlined in Figure $8 \mathrm{~B}$ ) of the questioned photograph are shown together in Figure 8C. A shared feature of these two extracted images is granular appearing shading. It appears the modified photograph had additionally been airbrush modified in the distal right neck. 
Citation: Burnett B (2016) Analysis of Fraudulent Photographs Submitted to Federal Court and the United States Congress. J Forensic Res 7: 343. doi: $10.4172 / 2157-7145.1000343$

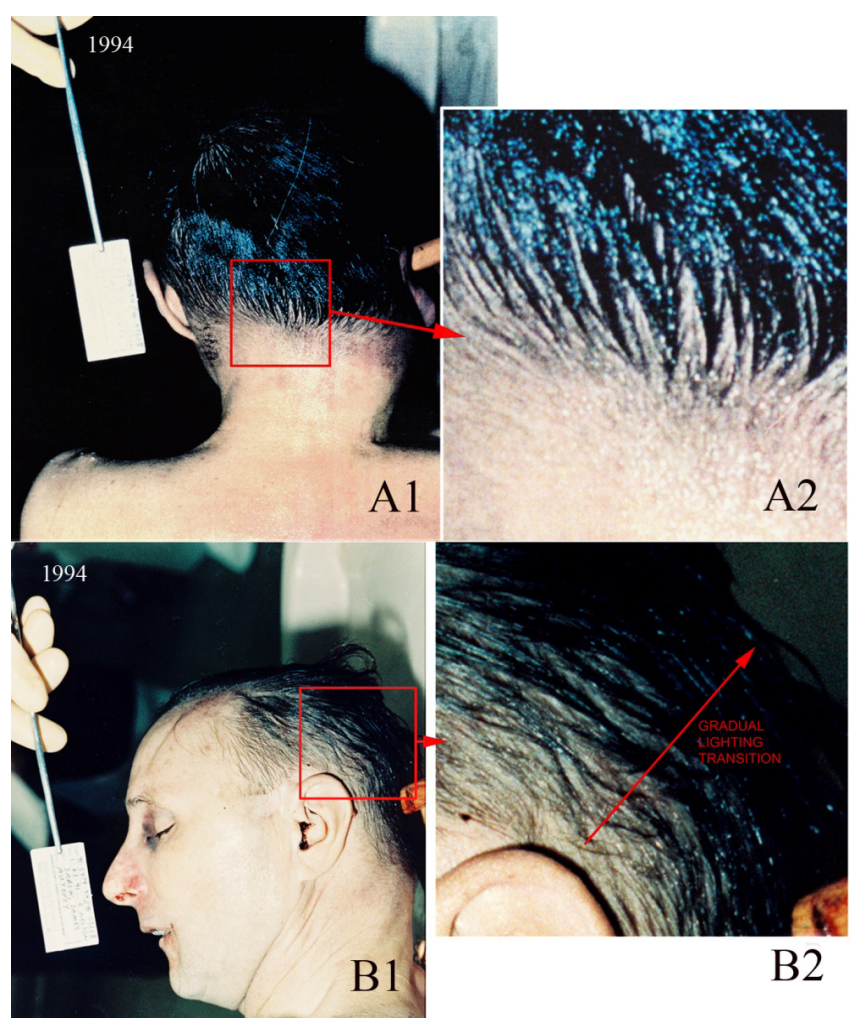

Figure 6: A1- The questioned image's hair at the hair line showing a lack of transition from the hair at the hairline to the hair on the rest of the head. A2Enlargement of boxed area. B1- Left side of the Colonel's head. B2- Enlarged area of the square in B1 showing the transition of the hair in the image to reflective (white) bands; image gamma adjusted by Photoshop's Levels.

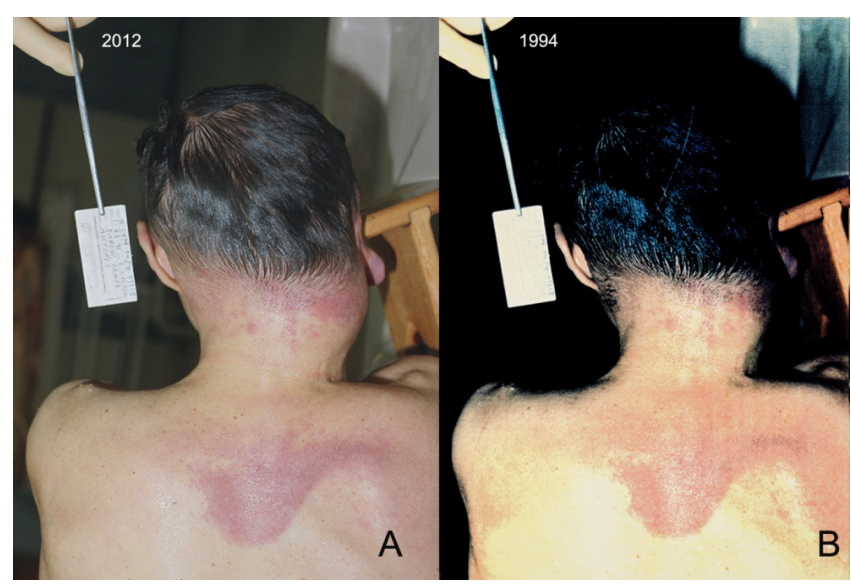

Figure 7: A- The photograph from the Orange County Coroner of the autopsy photograph upon which the modified photograph was made, B- Image of the modified photograph for comparison.

\section{Evidence of scanning the modified photograph}

The deep shadow areas of the questioned photograph have fine parallel horizontal lines or stripes when lightened by increasing gamma (Figure 9A). In lighter regions of the photograph, parallel stripes were also apparent (Figures 9B and 9C) and some were perpendicular to the stripes discovered in the deep shadow areas. Similar stripes have

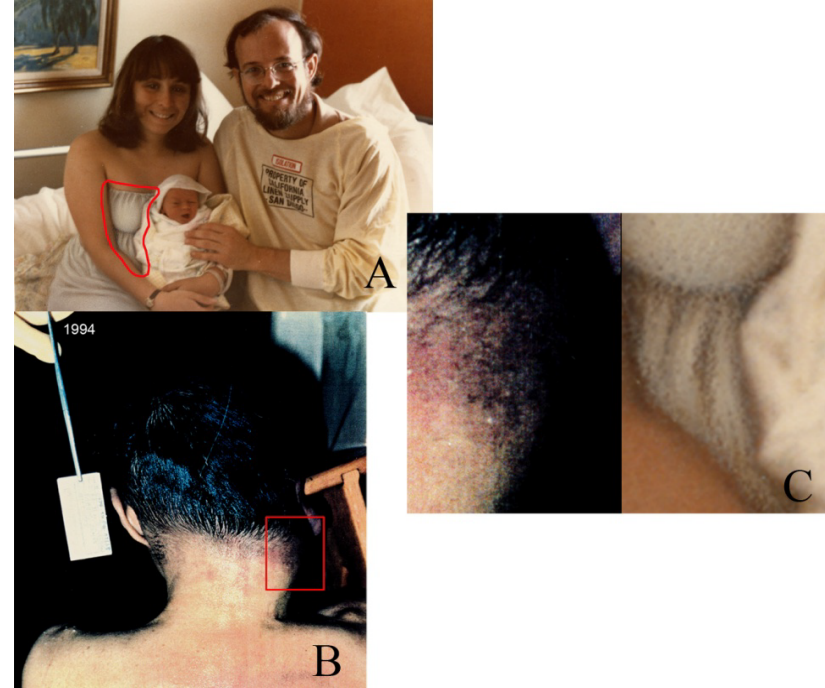

Figure 8: A- A family photograph (1981) that was airbrush-modified in the region outlined in red, B- The modified autopsy photograph; the red outline region is the area of probable airbrushing, C- Enlargements of the outline areas in A (right) and B (left); the known airbrushed example (right) shows a granular shading generated by this technique. A similar granular shadow area in the modified photograph (left) was likely created by airbrush. This is in contrast to a smooth transition to the deep shadow seen in unmodified photographs. Faint vertical scan lines can also be seen in the left image (see text).

been described by Russ [5, p. 31 therein] as a common defect of flatbed scanners of this period (early 1990s). Two facts will dispel the argument that these parallel stripes originated from the Epson scanner used to produce digital images of most of the photographs for this study. First, none of the other photograph scans from this scanner shows these stripes and second, a scan in 2013 with a commercial scanner of a negative made of the questioned photograph in 1994 shows identical stripe defects (Figure 9C).

Fabrication of the modified photograph: The results of this analysis indicate the questioned photograph was manipulated using a photo enlarger with advanced darkroom techniques. After the darkroom modification and airbrushing, the manipulated photograph was scanned at high resolution and then printed to a $35 \mathrm{~mm}$ negative followed by printing on standard photographic paper. A detailed reconstruction of how this photograph was made:

1. The original negative was printed (Figure 10, left) via an enlarger. An acetate sheet placed over the print. The mask was made of the area of the victim's upper back and neck by airbrush and the hairline was carefully detailed (Figure 10, middle). A new photograph print was made with the overlying mask in place. After the background and head were overexposed on the print, the mask was removed and additionally exposed for a much shorter time creating a print (Figure 10, right) quite different from the original photograph. The areas of the photograph that lacked mask coverage were heavily overexposed obliterating background detail and most detail of the head. Some of the hair (reflective due to being wet) was either light enough or was detailed on the mask to show in the head area of the modified photograph.

2. In order to complete the modification to remove the evidence of swelling on the right neck, airbrushing was likely used. An 
Citation: Burnett B (2016) Analysis of Fraudulent Photographs Submitted to Federal Court and the United States Congress. J Forensic Res 7: 343. doi: $10.4172 / 2157-7145.1000343$

Page 6 of 8

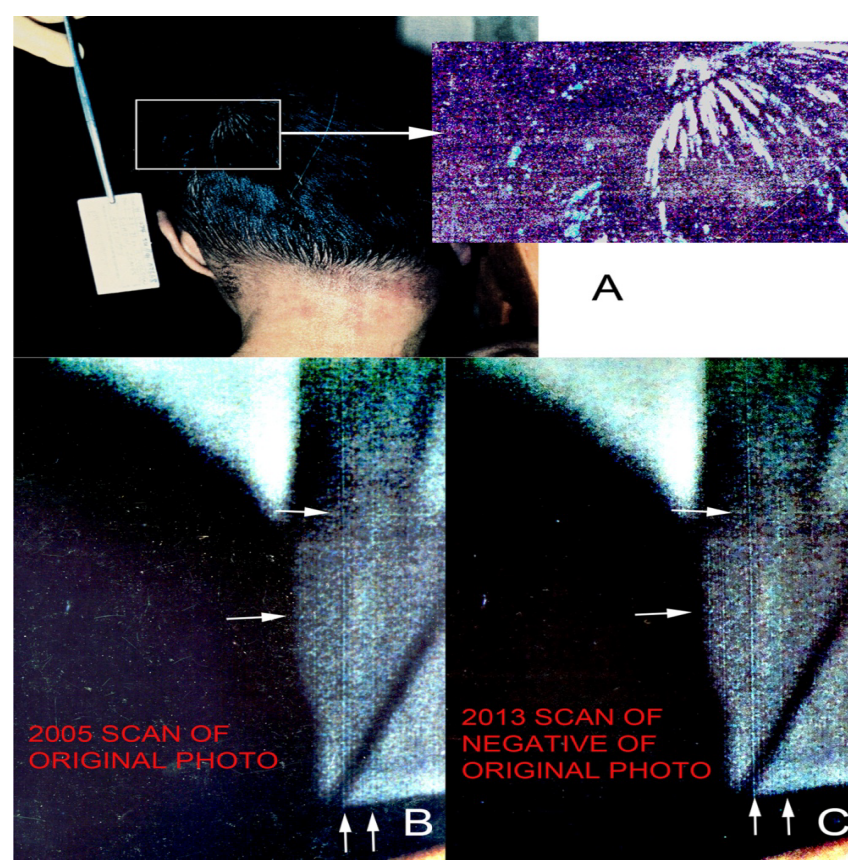

Figure 9: A- Horizontal lines or stripes within the deep shadow of the questioned photograph; the image on the right was gamma increased to show the stripes extend from the background into the head area of the victim. B, C- Comparisons of different scans of the modified photograph showing identical vertical and horizontal parallel stripes; from the upper right area of the questioned photograph. These images were maximally sharpened in Photoshop to accentuate the stripes. The stripes were not observed in any of the other autopsy photograph-images, regardless of source or generation. In $\mathrm{B}$, the arrows point to particularly prominent horizontal and vertical stripes. The image in $\mathrm{C}$ is from a scan of a negative of the questioned photograph print made by a commercial negative scanner.
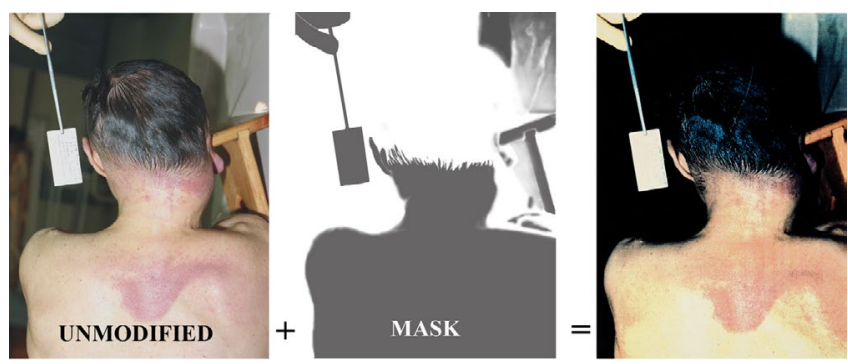

Figure 10: Reconstruction of the modified autopsy photograph using a mask. The exposure of the print from the original negative was long enough to obliterate any background and most head detail. The final exposure of the print occurred with the removal of the mask.

example of a known airbrush-modified photograph is shown in Figure 8A. The area of likely airbrush modification is outlined. Note in the enlargement of the demarked area (Figure 8C, right), the shadowing of the airbrush-modified area of the example photograph is not a uniform (as would be observed in an unmodified photograph) but granular. The area of probable airbrushing in the distal right neck of the modified photograph (Figure 8C, left) shows a similar granular feature.

3. The completed modified photograph (Figure 10, right) was then scanned as noted above producing the fine parallel stripes in the photograph. This image was then printed to a $35 \mathrm{~mm}$ negative strip which had the other autopsy photographs, thus maintaining the negative numbering on the negative strip. (Negative printers existed in the early 1990s.) Unfortunately, the plaintiffs in the Federal civil case did not request copies of the negatives of the autopsy photographs. Careful examination of the entire modified photograph shows the scan stripes exist throughout the photograph. If there was image processing, the stripes would have been disrupted, especially in the distal right neck area. There is no evidence of image processing.

The lighting on the back of the victim's head is inconsistent with the location of the light source (compare Figure s 5A1 and 5B1). None of the deep shadow background as well as most of the head in the original photograph (Figure 5A1) was preserved when the deep shadow of the modified photograph is increase to reveal structure content (Figure 5A2). Other autopsy photographs have structure content (Figure 4) within their deep shadows.

\section{The second modified photograph}

The DOD, as a result of the 2004 Congressional Authorization Act, was mandated to reinvestigate the death of the victim. Dr. Jon Nordby was retained and in late 2004 produced an 83-page report [2] in Adobe PDF format (release by the DOD in 2012 as a result of the Freedom of Information Act). As support for the alleged lack of neck swelling, Nordby selected a photograph similar to the modified photograph (Figure 11A, right). For analysis in this study, the image was extracted from the PDF file (Figure 11B) which was not obvious in the image shown in the Nordby document (Figure 11A). The original photograph (Figure 11C) is presented beside the extracted modified photograph (Figure 11B) for comparison. Although similar to the modified photograph of 1994 (Figure 2), the extracted image is different in that the right regions of the neck and right pinna have a deep red cast (Figure 11B, at arrow).

\section{Conclusion}

The region targeted by the modification in the original photograph (Figure 7A) is the right proximal occipital area of the head and the distal right neck (Figure 12A). The modification was done to make this region appear "normal" in the modified 1994 photograph (Figure 12B). It appears for the modified photograph used by Nordby [2], the first (1994) modified photograph was not available and a second modified photograph (Figure 11B) was constructed to appear identical to the first modified photograph (Figure 2). However, the right proximal head and neck in the first modified photograph (Figure 12B) is different from the second modified photograph (Figure 12C). This area in the second modified photograph is also deep red, which might have occurred with the photograph's PDF conversion for the Nordby report. The low resolution of the image of the second modified photograph, JPEG artifact rectangles and image modification by clipping of the blacks by Adobe Acrobat to PDF format prevent further analysis. The first questioned photograph (Figure 3) has many features which are inconsistent with the pathology report and other autopsy images. The analysis shows this photograph was constructed to deceive. The second questioned photograph (Figure 11B), used in a report to the U.S. Congress [2], is also modified from the original (Figure 11C), sharing many of the features of the first. It was constructed at a different time likely using image processing.

The modified photographs were constructed to hide the massive swelling on the right posterior head and neck, the first modified photograph by darkroom/airbrushing processing of this area (Figures 
Citation: Burnett B (2016) Analysis of Fraudulent Photographs Submitted to Federal Court and the United States Congress. J Forensic Res 7: 343. doi: $10.4172 / 2157-7145.1000343$

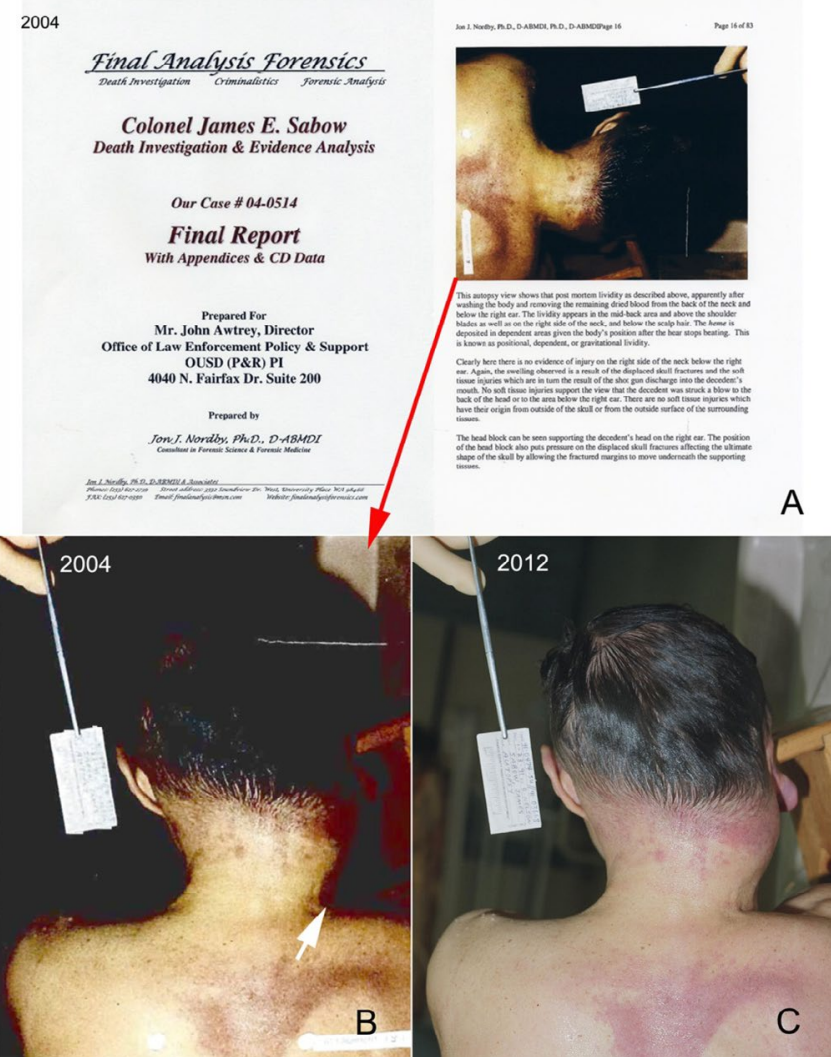

Figure 11: A. The title page and apparent modified image similar to the one shown in Figure 3; this document was submitted to the US Congress in 2004 [2]. The copy of the report received for this analysis was in PDF format. B. The image extracted from the PDF formated document. White arrow points to region of focused modification. C. Image of the original photograph sized and cropped for comparison to $\mathrm{B}$.

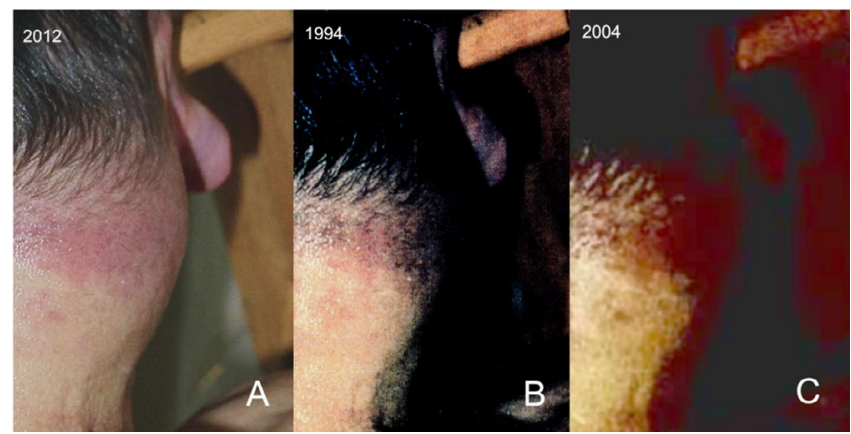

Figure 12: Hiding the neck swelling. A. The right proximal occipital area and neck from the Orange County Coroner's autopsy photographs (Figs. 8A and 9C) showing the extraordinary swelling in this area of the victim. B. A heavily modified photograph from the same area in the discovery released from the DOD in 1994. This shows an overt attempt to hide the swelling. C. An image of the same area (from the image shown in figure 12B) of the Nordby report to the US Congress; the dark red area was not apparent until this image was extracted from the Nordby PDF file. JPEG rectangle artifacts are present.

8 and 10 ) and the second modified photograph (Figure 11B likely by image processing. There is no detail within the deep shadow or black surrounding the head in the first modified photograph (Figure 5A2). The illumination on the back of the head by a flash is inconsistent with the topography of the upper back, neck and head (Figure 5). The same

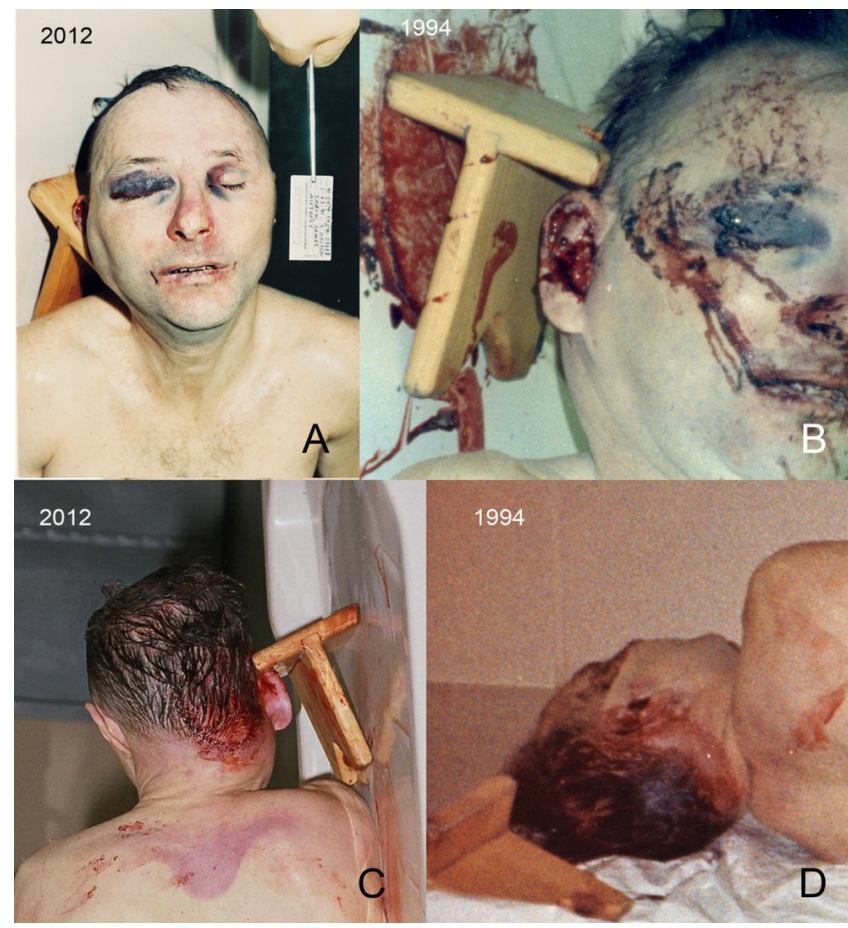

Figure 13: Other autopsy images showing the extraordinary swelling on the right side of the victim's head. A. Photograph of the anterior head and upper body after the cleaning in preparation for autopsy. B. Photograph of the right side of the Colonel's face prior to cleaning. C. Photograph of the posterior upper body of the victim showing dried blood surrounding (outlining) the swelling right occipital area of the head. D. Photograph of the posterior head and upper body prior to cleaning; the swelling of the right occipital region of the head is readily seen.

applies to the second modified photograph (Figure 11B). The second modified photograph, which was extracted from an Adobe Acrobat PDF file, is limited in its analysis to the non-blackened areas.

The modified autopsy photographs were not identified as such until December 2011, almost sixteen years after release of the first modified photograph which attests to the skill of its manufacture. Perhaps the second modified photograph used by Dr. Nordby [2] was instrumental in his coming to the likely incorrect conclusion as to the mode of the victim's death.

Curiously, other autopsy photographs of the victim show prominent features of blunt force trauma to the occipital area of the head. These photographs (Figure 13) show no evidence of manipulation.

\section{Acknowledgements}

Many thanks to Drs. John C. Russ (North Carolina State University, Raleigh, North Carolina) and Marcus Borengasser (University of Colorado, Boulder, Colorado) for their valuable comments. Permission to use the autopsy images in this publication by Dr. David Sabow, brother of Colonel James E. Sabow.

\section{References}

1. Singhania, A (1991) Autopsy record: Sabow, James Emery. Case Number 9100474-SU. Orange County Sheriff-Coroner, California, USA.

2. Nordby J (2004) The shotgun death of Col. James Sabow. United States Department of Defense, Washington, USA.

3. Posner JB, Saper CB, Schiff N, Plum F (2007) Plum and Posner's diagnosis of stupor and coma. Oxford University Press, Newyork, USA.

4. http://www.buzzle.com/articles/basilar-skull-fracture.html 
Citation: Burnett B (2016) Analysis of Fraudulent Photographs Submitted to Federal Court and the United States Congress. J Forensic Res 7: 343. doi: 10.4172/2157-7145.1000343

Page 8 of 8

5. Russ JC (1995) The Image Processing Handbook. (6 ${ }^{\text {nd }}$ edn.), CRC Press, Boca Raton, Florida, USA.
6. Sabow JD, Burnett BR (2010) The distinction between instantaneous and sudden death and how it is critical in equivocal death investigations. 